\title{
A nonlinear stability analysis of pattern formation in thin liquid films
}

\author{
EMILY M. TIAN ${ }^{\dagger}$ \\ Department of Mathematics and Statistics, Wright State University, \\ 3640 Colonel Glenn Hwy, Dayton, OH 45435-0001, USA \\ AND \\ DAVID J. WOLLKIND \\ Department of Mathematics, Washington State University, Pullman, WA 99164-3113, USA
}

[Received 14 December 2001 and in revised form 27 September 2002]

\begin{abstract}
The development of spontaneous stationary equilibrium patterns in thin liquid films is investigated by means of a hexagonal-planform weakly nonlinear stability analysis applied to the appropriate governing evolution equation for this phenomenon. In the long-wavelength limit the mathematical system modeling the liquid film can be reduced to such a single nonlinear partial differential timeevolution equation describing the layer thickness on an unbounded two-dimensional spatial domain and including the effects of gravity, intermolecular forces, and temperature-dependent surface tension. The main result of this analysis is that supercritical equilibrium patterns can occur for an interval of mean layer thickness with subcritical rupture occurring outside that interval. These patterns consist of surface ridges and hexagonal network-like cells or close-packed configurations of nanodroplets separated by relatively flat ultra thin films. In particular those morphological phase separation patterns are generated by the coupling between the long-range attractive and short-range repulsive intermolecular forces with cells being stable for the thicker layers; nanodroplets, for the thinner ones; and ridges, for layers of intermediate thickness. These theoretical predictions are in accord with both relevant experimental evidence involving thin liquid polymer, crystal, and metal films coating a solid substrate and numerical simulations of similar model equations as well as being consistent with dewetting-type rupture occurring for such situations by hole formation in relatively thick layers but by drop formation in thinner ones.
\end{abstract}

Keywords: Thin liquid films; nonlinear stability analysis; hexagonal pattern formation; morphological phase separation.

\section{Introduction and formulation of the problem}

Rayleigh-Bénard buoyancy-driven convection has to date provided perhaps the best studied example of nonlinear pattern selection (reviewed by Koschmieder [10]). One of the methods traditionally used to predict such pattern selection is a weakly nonlinear stability analysis that, although incorporating the nonlinearities of the relevant model system, basically pivots a perturbation procedure about the critical point of linear stability theory (reviewed by Wollkind et al. [32]). The advantage of such an approach over strictly numerical procedures is that it allows one to deduce quantitative relationships between system parameters and stable patterns which are

\footnotetext{
†Email: etian@math.wright.edu
}

${ }^{\ddagger}$ Email: dwollkind@wsu.edu 
valuable for experimental design and difficult to accomplish using simulation alone. Recently, there has been considerable interest generated in pattern formation and selection during the controlled plane-front solidification of a dilute binary alloy under the influence of an imposed temperature gradient and during chemical reactions occurring in an open gel continuously fed unstirred tank reactor. In order to predict the sequence of interfacial morphologies and Turing patterns actually observed during such solidification and chemical reactions, respectively, Wollkind et al. [33] and Wollkind and Stephenson [34] performed the same weakly nonlinear stability analysis as originally developed by Segel [20] to study Bénard convection cells on the governing systems of diffusion equations appropriate for modeling these phenomena. In particular all those investigations employed a hexagonal-planform weakly nonlinear stability analysis to determine the relevant parameter range for the transition between one-dimensional and hexagonal pattern formation.

We wish to continue our examination of nonlinear phenomena by investigating spontaneous pattern formation in thin liquid films. In this case an appropriate governing mathematical system can be reduced to a single nonlinear partial differential time-evolution equation describing the layer thickness on an unbounded two-dimensional spatial domain and including the effects of gravity, intermolecular forces, and temperature-dependent surface tension. We shall perform a hexagonalplanform weakly nonlinear stability analysis on that model equation and then compare the results obtained with both relevant experimental evidence and numerical simulations as well as place them in the context of some recent pattern formation studies. We begin below with a brief description of the phenomenon, a sketch of the reduction procedure required to derive the model equation, and a discussion of the methodology to be employed.

Liquid layers thinner than about $1 \mu \mathrm{m}\left(\equiv 10^{-6} \mathrm{~m}\right)$ are often unstable and spontaneously form various stationary equilibrium morphologies including surface ridges which exhibit a clearly defined critical wavelength and hexagonal cellular patterns or uniform distributions of nanodroplets separated by very thin films as depicted in the photographic reproductions of Sharma and Reiter [24], Xie et al. [36], Reiter [18, 19], and Herminghaus et al. [5]. These experiments involved thin liquid polymer, crystal, or metal films coating a solid substrate that were open above to the ambient air. Governing equations for situations of this sort have been developed by Williams and Davis [30], Sharma and Ruckenstein [25], Kheshgi and Scriven [9], Mitlin [13], Sharma and Jameel [22], Jameel and Sharma [6], Mitlin and Petviashvili [14], Khanna and Sharma [8], and Sharma and Khanna [23]. More generally the long-scale evolution of thin liquid films has been reviewed recently by Oron et al. [16].

Consider a thin liquid layer of mean thickness $h_{0}$ bounded from below by a planar solid surface located at $z=0$ and from above by an interface satisfying $z=h(x, y, \tau)$ which separates it from a passive gas (see Fig. 1). The liquid layer is assumed thin enough so that intermolecular forces must be taken into account but thick enough so that a continuum mechanical approach will still be valid. Then in the long-wavelength limit the Navier-Stokes and continuity equations for the liquid layer are given by

$$
\left(p+\rho_{0} g z+\phi\right)_{z}=P_{z}=0, \quad \mu u_{z z}=P_{x}, \quad \mu v_{z z}=P_{y}, \quad u_{x}+v_{y}+w_{z}=0 ; \quad(1.1 \mathrm{a}, \mathrm{b}, \mathrm{c}, \mathrm{d})
$$

with no-slip and no-penetration boundary conditions at the solid surface

$$
u=v=w=0 \quad \text { at } z=0
$$

and balance of the tangential and normal components of momentum and the kinematic boundary 


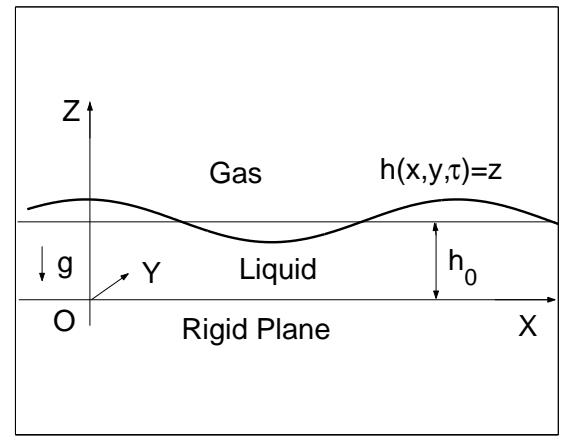

FIG. 1. The physical configuration showing the dimensional Cartesian coordinate system.

condition at the interface

$$
\mu u_{z}=\gamma_{x}, \mu v_{z}=\gamma_{y}, p_{0}-p=\gamma\left(h_{x x}+h_{y y}\right), h_{\tau}+u h_{x}+v h_{y}=w \text { at } z=h . \quad(1.3 \mathrm{a}, \mathrm{b}, \mathrm{c}, \mathrm{d})
$$

Here $\tau$ is time and the Cartesian coordinate system $(x, y, z)$ with corresponding velocity components $(u, v, w)$ has been employed while $\mu$ and $\rho_{0}$ are the constant shear viscosity and density of the Newtonian fluid liquid layer. In addition $g$ is the acceleration due to gravity while $p$ and $p_{0}$ refer to the pressure in the liquid layer and passive gas, respectively. Further, $\phi=\phi(h)$ and $\gamma=\gamma(h)$ are the intermolecular body force potential per unit volume and interfacial surface tension coefficient per unit length, both of which depend on the layer thickness $h$, the latter dependence being due to a nonzero $\gamma^{\prime}(h)$ caused by the Marangoni effect (see below). Basically the longwavelength asymptotic procedure [30] entails introducing a nondimensional scaling parameter $k>0$ such that $x, y, \tau, w=O(k) ; z, u, v, h=O(1) ; P, \gamma^{\prime}=O(1 / k)$; and $\gamma=O\left(1 / k^{3}\right)$. Then taking the limit as $k \rightarrow 0$ in the full system of partial differential equations and boundary conditions, one retains only those terms appearing in (1.1)-(1.3).

Solving equations (1.1) and boundary conditions (1.2) and (1.3a,b) for the velocity components, we obtain

$$
\begin{gathered}
\mu u=P_{x}\left(z^{2} / 2-h z\right)+\gamma_{x} z, \quad \mu v=P_{y}\left(z^{2} / 2-h z\right)+\gamma_{y} z, \\
\mu w=-\left(P_{x x}+P_{y y}\right) z^{3} / 6+\left[\left(P_{x} h\right)_{x}+\left(P_{y} h\right)_{y}-\left(\gamma_{x x}+\gamma_{y y}\right)\right] z^{2} / 2,
\end{gathered}
$$

which upon subsititution into the kinematic boundary condition (1.3d) yields the result

$$
3 \mu h_{\tau}-\left(h^{3} P_{x}\right)_{x}-\left(h^{3} P_{y}\right)_{y}+3\left[\left(h^{2} \gamma_{x}\right)_{x}+\left(h^{2} \gamma_{y}\right)_{y}\right] / 2=0 \quad \text { at } z=h .
$$

Finally using the definition of $P$ from (1.1a) in conjunction with (1.3c), we can represent (1.5a) in the higher dimensional form

$$
3 \mu h_{\tau}+\nabla \cdot\left[h^{3} \nabla\left\{\gamma(h) \nabla^{2} h\right\}\right]-\nabla \cdot\left[h^{3}\left\{\phi^{\prime}(h)+\rho_{0} g\right\} \nabla h\right]+3 \nabla \cdot\left[h^{2} \gamma^{\prime}(h) \nabla h\right] / 2=0
$$

where $\nabla$ denotes the two-dimensional gradient operator $(\partial / \partial x, \partial / \partial y)$ and $\nabla^{2} \equiv \nabla \cdot \nabla$.

We complete our formulation of this problem by letting [13]

$$
\phi(h)=a / h^{3}-b / h^{9}, \quad a, b>0,
$$


where the terms proportional to $a$ and $b$ in (1.6a) are due to the long range Van der Waals attraction and extremely short range Born repulsion, respectively, and

$$
\gamma(h)=\Gamma(T)=\gamma_{0}-\gamma_{1}\left(T-T_{0}\right) \quad \text { at } z=h, \quad \gamma_{0,1}>0,
$$

where consistent with the long-wavelength approximation the temperature $T$ satisfies

$$
T_{z z}=0 \text { for } 0<z<h, \quad T=T_{0}>0 \text { at } z=0, \quad T_{z}=-m_{0} \leqslant 0 \text { at } z=h .
$$

Here we are assuming that the planar solid surface is a perfect conductor maintained at a fixed temperature $T_{0}=O(1)$ while the interface is a poor conductor with an imposed heat flux $m_{0}=$ $O$ (1) to the environment [3]. Then defining $h_{c}$ by $\phi^{\prime}\left(h_{c}\right)=0$ and solving (1.7) for $T$ we find that

$$
b=a h_{c}^{6} / 3 \quad \text { and } \quad T=T_{0}-m_{0} z,
$$

which upon substitution into (1.6) yields

$$
\phi(h)=\left(a / h_{c}^{3}\right)\left[\left(h_{c} / h\right)^{3}-\left(h_{c} / h\right)^{9} / 3\right] \quad \text { and } \quad \gamma(h)=\gamma_{0}+\gamma_{1} m_{0} h,
$$

where the $a=O(1 / k)$ in (1.9a) is related to the Hamaker constant and $\gamma_{0}=O\left(1 / k^{3}\right), \gamma_{1}=$ $O(1 / k)$ in $(1.9 \mathrm{~b})$ are the capillarity and thermal Marangoni coefficients, respectively. Now incorporating the functions of (1.9) into (1.5b) and nondimensionalizing the resulting equation by introducing the scale factors $\rho_{0} h_{c}^{2} / \mu$ for time and $h_{c}$ for both length and layer thickness, we obtain

$$
h_{\tau}+S \nabla \cdot\left[h^{3} \nabla\left(\nabla^{2} h\right)\right]+A \nabla \cdot\left[\left(h^{-1}-h^{-7}\right) \nabla h\right]-G \nabla \cdot\left(h^{3} \nabla h\right)+M \nabla \cdot\left(h^{2} \nabla h\right) / 2=0
$$

where

$$
S=\frac{\gamma_{0} \rho_{0} h_{c}}{3 \mu^{2}}, \quad A=\frac{a \rho_{0}}{\mu^{2} h_{c}}, \quad G=\frac{g \rho_{0}^{2} h_{c}^{3}}{3 \mu^{2}}, \quad M=\frac{\gamma_{1} m_{0} \rho_{0} h_{c}^{2}}{\mu^{2}},
$$

and a second term proportional to $M, \nabla \cdot\left[h^{3} \nabla\left(h \nabla^{2} h\right)\right]$, has been neglected in (1.10a) since it is negligible with respect to the one retained by virtue of the long-wavelength approximation. Finally we introduce the rescaled variables [30]

$$
t=A^{2} \tau / S, \quad\left(r_{1}, r_{2}\right)=(A / S)^{1 / 2}(x, y), \quad H\left(r_{1}, r_{2}, t\right)=h(x, y, \tau),
$$

which transforms (1.10) into

$H_{t}+\nabla_{2} \cdot\left[H^{3} \nabla_{2}\left(\nabla_{2}^{2} H\right)\right]+\nabla_{2} \cdot\left[\left(H^{-1}-H^{-7}\right) \nabla_{2} H\right]-\varepsilon \nabla_{2} \cdot\left(H^{3} \nabla_{2} H\right)+\beta \nabla_{2} \cdot\left(H^{2} \nabla_{2} H\right)=0$

where

$$
\varepsilon=\frac{G}{A}=\frac{\rho_{0} g h_{c}^{4}}{3 a}>0, \quad \beta=\frac{M}{2 A}=\frac{\gamma_{1} m_{0} h_{c}^{3}}{2 a} \geqslant 0,
$$

and $\nabla_{2} \equiv\left(\partial / \partial r_{1}, \partial / \partial r_{2}\right)$.

This is the model spatio-temporal evolution equation we wish to analyze for the type of interfacial morphologies catalogued earlier. Although bifurcation analyses relevant to the linear problem and numerical simulations relevant to the nonlinear one were conducted on evolution equations of that sort in the references already cited, except for Sharma and Khanna [23] their primary emphasis was on the related phenomenon of thin film rupture and none of them included 
all of the features contained in (1.10). Specifically, Williams and Davis [30] performed a numerical simulation on a one-dimensional version of (1.12) with $\varepsilon=\beta=0$ in the absence of short-range repulsive forces while Sharma and Ruckenstein [25] and Kheshgi and Scriven [9] performed similar analyses on that equation with $\varepsilon=0$ or $\beta=0$, respectively, where in the former instance the Marangoni effect was due to surfactant concentration. Then Sharma and Jameel [22] and Jameel and Sharma [6] performed both a linear stability analysis and a numerical simulation on a onedimensional version of an equation similar to (1.12) but with $\varepsilon=\beta=0$ and the repulsive force term in (1.6a) taken proportional to $\exp \left(-h / \ell_{0}\right)$ for a prescribed correlation length $\ell_{0}$. Sharma and Khanna [23] extended this numerical simulation to two-dimensions. Mitlin [13] and Mitlin and Petviashvili [14] also performed both a linear stability analysis and a numerical simulation on a one-dimensional version of (1.12) with $\beta=0$. Khanna and Sharma [8] extended this numerical simulation to two-dimensions with $\varepsilon=0$ as well. Very recently Oron and Bankoff [15] performed both a weakly nonlinear stability analysis and a numerical simulation on a one-dimensional version of an evolution equation similar to (1.12) but with $\varepsilon=0$, our thermal Marangoni effect replaced by one appropriate for more general heat transfer, and the repulsive force term of (1.6a) taken proportional to $h^{-4}$ rather than to $h^{-9}$. In particular they felt that such a term was superior to one proportional to $h^{-9}$ for representing actual substrates which are often coated or microscopically rough since the latter Lennard-Jones Born potential had been deduced for an idealized clean smooth surface. We shall defer a discussion of this matter until the comparisons included in our last section.

To date no two-dimensional weakly nonlinear stability analysis has been performed on any of the thin liquid film lubrication-type evolution equations described above. In order to alleviate this deficiency we wish to perform a hexagonal-planform weakly nonlinear stability analysis on (1.12). Toward this end we note that there exists a planar interface solution

$$
H_{0}(\alpha)=\alpha=h_{0} / h_{c}>0
$$

of (1.12a) which satisfies the implicit far-field condition

$$
H \text { remains bounded as } r_{1}^{2}+r_{2}^{2} \rightarrow \infty
$$

and represents a layer of uniform depth. It is the weakly nonlinear stability of this solution to onedimensional and hexagonal-planform perturbations with which we shall be concerned in Sections 2 and 3, respectively, the former being a special case of the latter.

\section{One-dimensional analysis: Linear and nonlinear stability results}

We first perform a one-dimensional analysis of (1.12) by considering a solution to it through third order terms of the form (see below for a justification of this truncation procedure)

$$
\begin{gathered}
H\left(r_{1}, r_{2}, t\right) \sim \alpha+A_{1}(t) \cos \left(q r_{1}\right)+A_{1}^{2}(t)\left[H_{20}+H_{22} \cos \left(2 q r_{1}\right)\right] \\
+A_{1}^{3}(t)\left[H_{31} \cos \left(q r_{1}\right)+H_{33} \cos \left(3 q r_{1}\right)\right]
\end{gathered}
$$

where the amplitude function $A_{1}(t)$ satisfies the Landau equation

$$
\frac{\mathrm{d} A_{1}}{\mathrm{~d} t} \sim \sigma A_{1}-a_{1} A_{1}^{3}
$$

and $q=2 \pi / \lambda, \lambda$ being the wavelength of the class of spatially periodic perturbations under investigation. Substituting the solution of (2.1) into (1.12), we obtain a sequence of problems, 
one for each pair of values of $m$ and $n$, which corresponds to a term of the form $A_{1}^{m}(t) \cos \left(n q r_{1}\right)$ appearing explicitly in (2.1a).

Then the linear stability problem for $m=n=1$ yields the secular equation

$$
\sigma=\left(\beta \alpha^{2}-\varepsilon \alpha^{3}+\alpha^{-1}-\alpha^{-7}\right) q^{2}-\alpha^{3} q^{4}=\sigma_{0}\left(q^{2} ; \beta, \varepsilon\right),
$$

from which we can deduce the stability criterion

$$
\beta \leqslant \beta_{0}(\alpha ; \varepsilon)=\varepsilon \alpha-\alpha^{-3}+\alpha^{-9} .
$$

When $(2.2 \mathrm{~b})$ is violated we arrive at the instability criterion

$$
\beta>\beta_{0}(\alpha ; \varepsilon) .
$$

Under this condition, after Sekimura et al. [21] who used an identical approach on a secular equation of the same form, we select a fixed value $\beta_{c}$ satisfying

$$
\beta>\beta_{c} \geqslant 0
$$

such that for

$$
\begin{gathered}
q^{2}=q_{c}^{2}=Q_{c}\left(\alpha ; \beta_{c}, \varepsilon\right)=\alpha^{-4}-\alpha^{-10}+\beta_{c} \alpha^{-1}-\varepsilon>0, \\
\sigma=\alpha^{2} Q_{c}\left(\alpha ; \beta_{c}, \varepsilon\right)\left(\beta-\beta_{c}\right)=\alpha^{3} Q_{c}^{2}\left(\alpha ; \beta_{c}, \varepsilon\right) / 4=\delta^{2}>0 .
\end{gathered}
$$

This scenario is depicted in Fig. 2a. There is some merit in our offering a more detailed explanation of the Sekimura et al. [21] approach at this point. It involves selecting a critical value $\beta_{c} \geqslant 0$ of the bifurcation parameter so that the associated parabolic dispersion relation $\sigma=\sigma_{0}\left(q^{2} ; \beta_{c}, \varepsilon\right)$ has its maximum which occurs at $q^{2}=q_{c}^{2} / 2$ satisfying $\sigma_{0}\left(q_{c}^{2} / 2 ; \beta_{c}, \varepsilon\right)=\delta^{2} \ll 1$ where the critical wavenumber $q_{c}^{2}>0$ is defined by $\sigma_{0}\left(q_{c}^{2} ; \beta_{c}, \varepsilon\right)=0$. Then the bifurcation parameter $\beta$ is perturbed around this critical value $\beta_{c}$ in such a way that the resulting dispersion relation $\sigma=\sigma_{0}\left(q^{2} ; \beta, \varepsilon\right)$ satisfies $\sigma_{0}\left(q_{c}^{2} ; \beta, \varepsilon\right)=\delta^{2}$. The constraint of (2.3b) or equivalently the instability criterion (2.2c) particularized to $\beta_{c}$ occurs whenever the nondimensional mean layer thickness $\alpha$ satisfies the spinodal-decomposition [2]-like condition

$$
\alpha_{1}<\alpha<\alpha_{2} .
$$

When $\beta_{c}=0$ as was true for the isothermal problem of Mitlin [13],

$$
\alpha_{1} \cong 1+\varepsilon / 6, \quad \alpha_{2} \cong \varepsilon^{-1 / 4} \quad \text { should } \varepsilon \ll 1,
$$

which is typically the case (see Fig. 2b). Given the representative values $[9,13]$

$$
\rho_{0} g=10^{4} \mathrm{~kg} /\left(\mathrm{m}^{2} \mathrm{sec}^{2}\right), \quad 3 a=10^{-21} \mathrm{~kg} \mathrm{~m} / \mathrm{sec}^{2}, \quad h_{c}=10^{-9} \mathrm{~m} \equiv 1 \mathrm{~nm},
$$

we find from (1.12) and (2.4b) that $\varepsilon=10^{-11}$ and $\alpha_{2} \cong 562$, for example. We plot the $\sigma$ of (2.3c) versus $\alpha$ for these representative values in Fig. 2c. Observe that for $\varepsilon=0, \alpha_{2} \rightarrow \infty$, which was Mitlin's [13] rationale for including that gravity effect. In what follows we shall equate the $q$ contained in (2.1a) to $q_{c}$ identically. We plot the $q_{c}^{2}$ of (2.3b) versus $\alpha$ for these same values in Fig. 2d. 
(a)

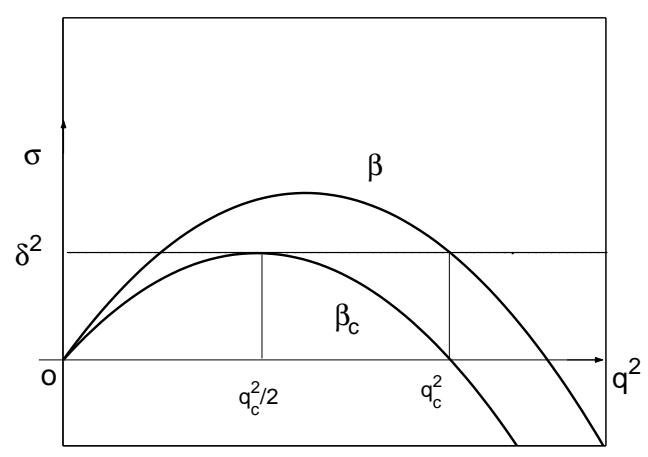

(c)

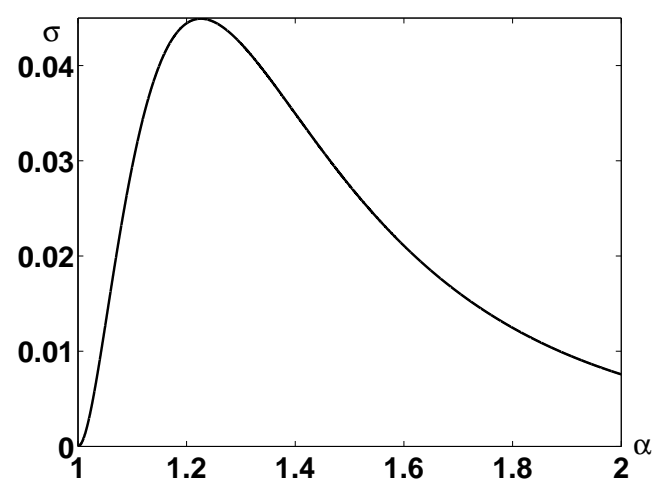

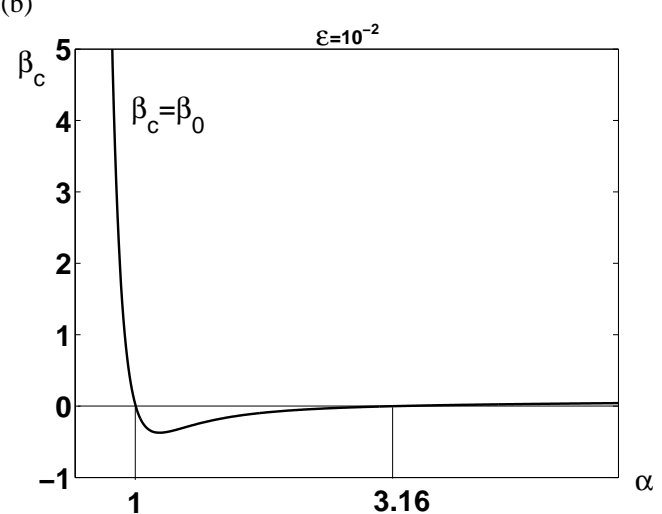

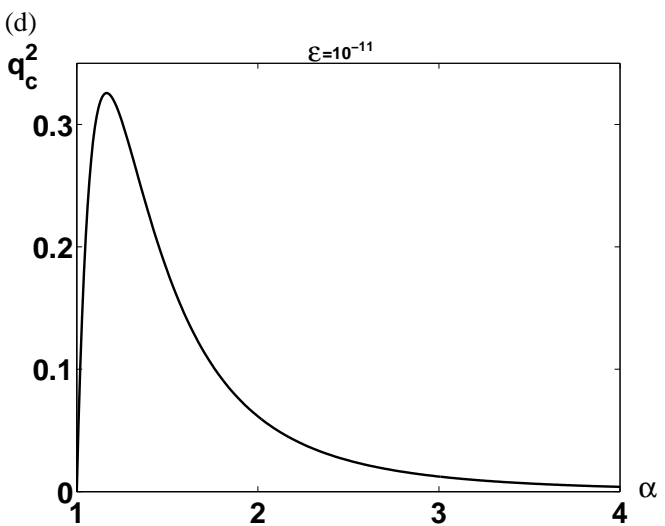

FIG. 2. (a) A schematic plot of (2.3) in the $q^{2}-\sigma$ plane. (b) The stability curve $\beta_{c}=\beta_{0}(\alpha ; \varepsilon)$ of $(2.2 \mathrm{~b}, \mathrm{c})$ for $\varepsilon=10^{-2}$. Note that its intercepts satisfy $\alpha_{1} \cong 1$ and $\alpha_{2} \cong \sqrt{10} \cong 3$.16. Plots of (c) $\sigma=\alpha^{3} Q_{c}^{2}\left(\alpha ; \beta_{c}, \varepsilon\right) / 4$ and (d) $q_{c}^{2}=Q_{c}\left(\alpha ; \beta_{c}, \varepsilon\right)$ of $(2.3 \mathrm{~b}, \mathrm{c})$ versus $\alpha$ for $\beta_{c}=0$ and $\varepsilon=10^{-11}$.

Continuing with our description of the results of this one-dimensional expansion procedure, the second-order problems corresponding to $m=2$ and $n=0$ or 2 can be solved in a straightforward manner to yield

$$
H_{20}=0, \quad H_{22}=\frac{10 \alpha^{-9}-4 \alpha^{-3}-\beta_{c}+2\left(\beta-\beta_{c}\right)}{2 \alpha^{2}\left[6 q_{c}^{2}+\left(\beta_{c}-\beta\right) / \alpha\right]} .
$$

Although there are also two third-order problems, it is permissible to concentrate our attention exclusively on the one corresponding to $m=3$ and $n=1$ which contains the Landau constant $a_{1}$ for the Fredholm-type method of solvability we shall use. That problem may be represented symbolically as

$$
\begin{aligned}
a_{1}-2 \sigma(\beta) H_{31}(\beta)= & \left(21 \alpha^{2} q_{c}^{2}+\alpha^{-2}-7 \alpha^{-8}+3 \varepsilon \alpha^{2}-2 \beta \alpha\right) q_{c}^{2} H_{22}(\beta) / 2 \\
& +q_{c}^{2}\left(3 \alpha q_{c}^{2}+28 \alpha^{-9}-\alpha^{-3}+3 \varepsilon \alpha-\beta\right) / 4
\end{aligned}
$$

Here we have only explicitly denoted the $\beta$-dependence of the quantities in question for ease of exposition. Now taking the limit of (2.7) as $\beta \rightarrow \beta_{c}$ or equivalently as $\delta \rightarrow 0$, employing (2.3) and 
(2.6), and assuming the requisite continuity at $\beta=\beta_{c}$, we obtain the solvability condition

$$
\begin{aligned}
a_{1}= & \left(22 \alpha^{-4}-28 \alpha^{-10}-18 \varepsilon+19 \beta_{c} \alpha^{-1}\right)\left(10 \alpha^{-9}-4 \alpha^{-3}-\beta_{c}\right) / 24 \\
& +\left(2 \alpha^{-3}+25 \alpha^{-9}+2 \beta_{c}\right)\left(\alpha^{-4}-\alpha^{-10}-\varepsilon+\beta_{c} \alpha^{-1}\right) / 4 .
\end{aligned}
$$

For the examination of the sign of $a_{1}$ to be presented below, it is convenient to rewrite the expression of (2.8) in the form

$$
a_{1}=c_{0}\left(\beta_{1}-\beta_{c}\right)\left(\beta_{c}-\beta_{2}\right) \quad \text { with } \quad c_{0}=7 \alpha^{-1} / 24
$$

where $\beta_{1,2}$ are the roots of the associated quadratic

$$
7 \beta^{2}-c_{1} \beta+c_{2}=0
$$

with

$$
c_{1}=356 \alpha^{-9}-74 \alpha^{-3}+6 \varepsilon \alpha, c_{2}=430 \alpha^{-18}-470 \alpha^{-12}+76 \alpha^{-4}+10 \varepsilon\left(33 \alpha^{-8}-6 \alpha^{-2}\right),
$$

and hence given explicitly by

$$
\beta_{1,2}(\alpha ; \varepsilon)=\left[c_{1} \pm\left(c_{1}^{2}-28 c_{2}\right)^{1 / 2}\right] / 14
$$

A main feature of the weakly nonlinear stability theory implicit to the formulation of our expansion (2.1) with $q \equiv q_{c}$ is a phenomenological interpretation of the problem under examination based upon the long-time behavior of the solution $A_{1}(t)$ to the truncated amplitude equation (2.1a). In general the dynamics of such a Landau equation with real coefficients can be divided into the four qualitatively different cases represented by the possibility of $\sigma$, its growth rate, and $a_{1}$, its Landau constant, being either positive or negative. These cases can be catalogued as follows:

Case (i): $\sigma, a_{1}>0$. There exists a stable equilibrium solution $A_{e}^{2}=\sigma / a_{1}$. Since $\sigma>0\left(\beta_{c}>\beta_{0}\right)$, linear theory would predict instability whereas our nonlinear analysis shows the existence of this finite amplitude supercritically stable equilibrium state.

Case (ii): $\sigma>0, a_{1}<0$. The undisturbed state $A_{1}=0$, which corresponds to a planar interface solution, is unstable in this case, as well; however now finite amplitude effects tend to enhance such disturbance growth.

Case (iii): $\sigma, a_{1}<0$. There exists an unstable equilibrium solution $A_{e}^{2}=\sigma / a_{1}$. This is an instance of a subcritical instability in that linear theory predicts stability of the planar interface solution to infinitesimal disturbances since $\sigma<0\left(\beta_{c}<\beta_{0}\right)$ whereas nonlinear theory shows it can be unstable to disturbances the amplitudes of which satisfy $A_{1}^{2}>\sigma / a_{1}$. Such an occurrence is often termed a metastable state.

Case (iv): $\sigma<0, a_{1}>0$. The planar interface solution is stable to both infinitesimal and finite amplitude disturbances.

From the description of the four cases just summarized we can see that the stability behavior of the Landau equation is crucially dependent upon the sign of $a_{1}$. Hence in order to determine this behavior it is necessary that we next examine the formula for $a_{1}$ given by (2.8) or equivalently by (2.9) and (2.10). Toward that end we plot the loci $\beta_{c}=\beta_{1,2}$ of (2.10) on which $a_{1}=0$ as well as the 
relevant portion of the marginal stability curve of linear theory $\beta_{c}=\beta_{0}$ of (2.2) in the $\alpha$ - $\beta_{c}$ plane of Fig. 3a for the $\varepsilon=10^{-11}$ of (2.5). From this figure in conjunction with (2.9), we observe that, for a given value of $\beta_{c}=\beta_{c}^{(0)} \geqslant 0, a_{1}>0$ whenever

$$
\alpha_{1}<\alpha_{3}<\alpha<\alpha_{4}<\alpha_{2}
$$

where

$$
\beta_{c}^{(0)}=\beta_{0}\left(\alpha_{1} ; \varepsilon\right)=\beta_{2}\left(\alpha_{3} ; \varepsilon\right)=\beta_{1}\left(\alpha_{4} ; \varepsilon\right)=\beta_{0}\left(\alpha_{2} ; \varepsilon\right) .
$$

Then $\alpha_{3}=1.02, \alpha_{4}=1.31 ; \alpha_{3}=0.93, \alpha_{4}=1.27 ; \alpha_{3}=0.80, \alpha_{4}=1.16$; should $\beta_{c}=0,1,7$; these $\beta_{c}$ corresponding to $\gamma_{1} m_{0} h_{c}=0,2 / 3,14 / 3$ dynes/cm, respectively, which are typical values for that parameter as employed by Sharma and Ruckenstein [25]. We demonstrate these results explicitly for the isothermal situation of Fig. 2c by graphing $a_{1}$ of (2.8) versus $\alpha$ in Fig. 3b with $\beta_{c}=0$ and $\varepsilon=10^{-11}$.

(a)

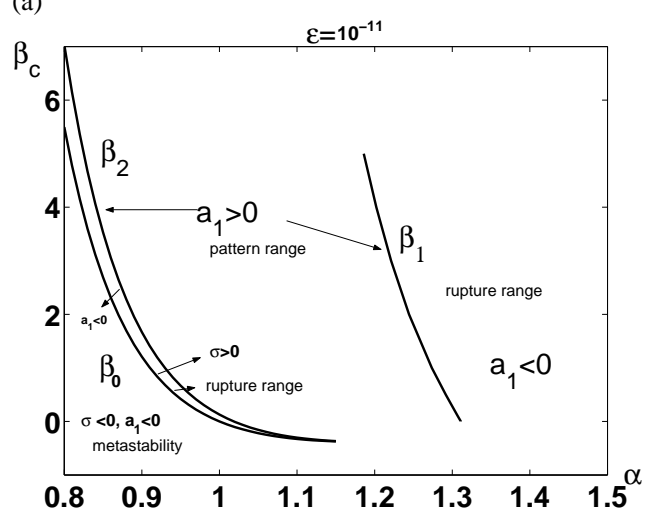

(b)

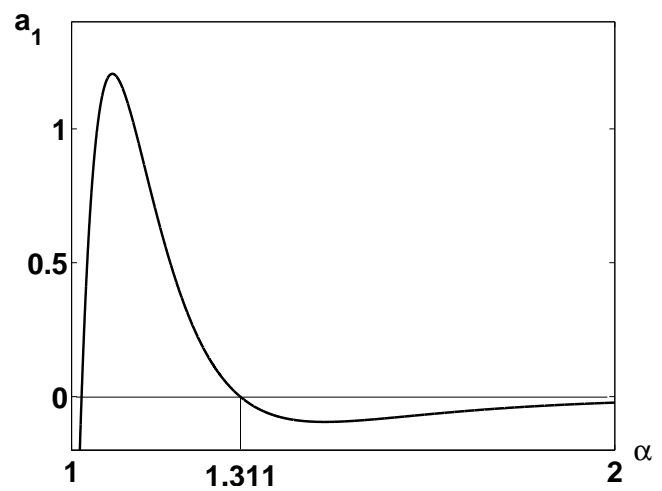

FIG. 3. (a) Plots of $\beta_{c}=\beta_{0}(\alpha ; \varepsilon)$ of $(2.2 \mathrm{~b}, \mathrm{c})$ and $\beta_{c}=\beta_{1,2}(\alpha ; \varepsilon)$ of (2.10) for $\varepsilon=10^{-11}$ explicitly denoting the relevant regions of $\alpha-\beta_{c}$ parameter space corresponding to the three possible cases represented by the growth rate $\sigma$ and the Landau constant $a_{1}$ being either positive or negative. Observe that the fourth case $\sigma<0, a_{1}>0$ cannot occur. (b) A plot of $a_{1}$ of (2.8) versus $\alpha$ for $\beta_{c}=0$ and $\varepsilon=10^{-11}$.

Having identified those regions in Fig. 3a corresponding to the cases catalogued above, we now offer a phenomenological interpretation of each of these cases.

Case (i). Since as can be seen from Fig. 3a, $\beta_{0}<\beta_{2}<\beta_{1}$ for $\alpha$ satisfying (2.11a), it follows that $\sigma, a_{1}>0$. Thus we can conclude that in this parameter range the instability of (2.3) represents a periodic one-dimensional equilibrium pattern consisting of stationary parallel liquid ridges separated by very thin films and having a characteristic wavelength of

$$
\lambda_{c}=2 \pi / Q_{c}^{1 / 2}\left(\alpha ; \beta_{c}, \varepsilon\right),
$$

in qualitative agreement with Oron and Bankoff's [15] supercritical prediction. For the isothermal problem of Figs. $2 \mathrm{c}$ and $3 \mathrm{~b}$ we can deduce that

$$
0<\sigma=\delta^{2} \leqslant .045, \quad 0<a_{1} \leqslant 1.2 ;
$$


and hence

$$
A_{e}^{2}=\sigma / a_{1} \cong \delta^{2}
$$

which implies

$$
\lim _{t \rightarrow \infty} H\left(r_{1}, r_{2}, t\right) \sim \alpha+\delta \cos \left(2 \pi r_{1} / \lambda_{c}\right) \quad \text { as } \delta \rightarrow 0
$$

and is a necessary requirement for the justification of the truncation procedure inherent to the asymptotic representation of (2.1) with $\lambda \equiv \lambda_{c}$. That truncation can be most easily accomplished by rewriting the full amplitude equation associated with (2.1b) in the form [32]

$$
\frac{1}{a_{1}} \frac{d A_{1}}{d t}=\frac{\sigma}{a_{1}} A_{1}-A_{1}^{3}+O\left(A_{1}^{5}\right),
$$

introducing the rescaled variables

$$
\eta=\sigma t, \quad \mathcal{A}(\eta)=\frac{A_{1}(t)}{\delta}
$$

employing (2.13b), cancelling the resulting common $\delta^{3}$ factor to obtain

$$
\frac{d \mathcal{A}}{d \eta}=\mathcal{A}-\mathcal{A}^{3}+O\left(\delta^{2}\right),
$$

and neglecting terms of $O\left(\delta^{2}\right)$ in (2.13f). Similarly the truncation inherent to (2.1a) results upon neglect of terms of $O\left(\delta^{4}\right)$ while retaining those through $O\left(\delta^{3}\right)$ for the full expansion. In this context we can deduce from Fig. $2 d$ that

$$
0<q_{c}^{2}<.325 \text { or } 0<q_{c}<.57
$$

and hence conclude $q_{c}=O(1)$ in essence for the $\alpha$-range of (2.11a) which is a consequence of the proper selection of scale factors and introduction of nondimensional variables in Section 1 [32].

Case (ii). For

$$
\alpha_{1}<\alpha<\alpha_{3} \text { or } \alpha_{4}<\alpha<\alpha_{2}
$$

the instability of (2.3) results in a dewetting-type rupture of the thin liquid film since $\sigma>0$ and $a_{1}<0$ [15] as indicated in Fig. 3a.

Case (iii). For

$$
\alpha<\alpha_{1} \quad \text { or } \quad \alpha>\alpha_{2}
$$

since $\sigma, a_{1}<0$ there is a subcritical instability or a metastable state. We note that numerical simulations of analogous equations in the absence of all intermolecular forces by Van Hook et al. [29] and in the absence of the short-range repulsive force and the Marangoni effect by Khesghi and Scriven [9] both resulted in rupture. Since our $a_{1}$ of (2.8) reduces to the asymptotic representations $-7 \alpha^{-1} \beta_{c}^{2} / 24$ and $-19 \alpha^{-7} / 6$, respectively, as $\varepsilon \rightarrow 0$ for these two cases, we also identify such subcritical $\left(a_{1}<0\right)$ behavior with thin film rupture [15].

Case (iv). Since the conditions (2.11a) and (2.14b), corresponding to $a_{1}>0$ and $\sigma<0$, respectively, are mutually exclusive this case can never occur. Hence the planar interface solution is always unstable for our thin liquid film problem. 


\section{Two-dimensional analysis: Hexagonal-planform stability results}

Wishing to refine the predictions of our one-dimensional analysis contained in Fig. 3a we next perform a two-dimensional analysis of (1.12) by seeking a hexagonal-planform solution of it which to lowest order satisfies

$$
\begin{aligned}
H(\mathbf{r}, t)-\alpha \sim & f\left(r_{1}, r_{2}, t\right)=A_{1}(t) \cos \left[q_{c} r_{1}+\phi_{1}(t)\right] \\
& +A_{2}(t) \cos \left[q_{c}\left(r_{1}-\sqrt{3} r_{2}\right) / 2-\phi_{2}(t)\right] \\
& +A_{3}(t) \cos \left[q_{c}\left(r_{1}+\sqrt{3} r_{2}\right) / 2-\phi_{3}(t)\right]
\end{aligned}
$$

where $\mathbf{r}=\left(r_{1}, r_{2}\right)$ and

$$
\begin{aligned}
\frac{\mathrm{d} A_{i}}{\mathrm{~d} t} & \sim \sigma A_{i}-4 a_{0} A_{j} A_{k} \cos \left(\phi_{i}+\phi_{j}+\phi_{k}\right)-A_{i}\left[a_{1} A_{i}^{2}+2 a_{2}\left(A_{j}^{2}+A_{k}^{2}\right)\right], \\
A_{i} \frac{\mathrm{d} \phi_{i}}{\mathrm{~d} t} & \sim 4 a_{0} A_{j} A_{k} \sin \left(\phi_{i}+\phi_{j}+\phi_{k}\right), \quad(i, j, k)=\text { even permutation of }(1,2,3) .
\end{aligned}
$$

In doing so we shall be closely following the approach of Wollkind and Stephenson [34] and Wollkind [31] for their chemical Turing pattern formation analyses. Hence it is our intention merely to sketch this methodology with a focus on the relevant stability results while observing that those references and Tian [28] contain the complete details of both the general procedure and its specific application to the thin liquid film problem, respectively. The weakly nonlinear stability behavior of the amplitude-phase equations $(3.1 \mathrm{~b}, \mathrm{c})$ to be described below depends only on the values of their growth rate and Landau constants. We can determine the solvability conditions for these Landau constants most easily by introducing the transformation [34]

$$
A_{2}(t)=A_{3}(t)=B_{1}(t) / 2, \quad \phi_{1}(t)=\phi_{2}(t)=\phi_{3}(t) \equiv 0
$$

which reduces (3.1) to

$$
f\left(r_{1}, r_{2}, t\right)=A_{1}(t) \cos \left(q_{c} r_{1}\right)+B_{1}(t) \cos \left(q_{c} r_{1} / 2\right) \cos \left(\sqrt{3} q_{c} r_{2} / 2\right)
$$

where

$$
\begin{aligned}
\frac{\mathrm{d} A_{1}}{\mathrm{~d} t} \sim \sigma A_{1}-a_{0} B_{1}^{2}-A_{1}\left(a_{1} A_{1}^{2}+a_{2} B_{1}^{2}\right) \\
\frac{\mathrm{d} B_{1}}{\mathrm{~d} t} \sim \sigma B_{1}-4 a_{0} A_{1} B_{1}-B_{1}\left[2 a_{2} A_{1}^{2}+\left(a_{1}+2 a_{2}\right) B_{1}^{2} / 4\right] .
\end{aligned}
$$

We note that the forms of the second- and third-order terms in the expansions of (3.1a) in conjunction with (3.2) and of (3.3a,b) can be deduced by examining the functional dependence of and the amplitude functions proportional to $\cos \left(q_{c} r_{1}\right)$ and $\cos \left(q_{c} r_{1} / r_{2}\right) \cos \left(\sqrt{3} q_{c} r_{2} / 2\right)$, respectively, in $f^{2}\left(r_{1}, r_{2}, t\right)$ and $f^{3}\left(r_{1}, r_{2}, t\right)$. Observe in this context that by taking $B_{1}(t) \equiv 0$ these expansions can be further reduced to those of (2.1) with $q \equiv q_{c}$ once we make the identification $H_{m n}=H_{m 0 N 0}$ for $N=2 n$ where the notation $H_{m k N \ell}$ is being employed to represent the coefficient of each higher order term in (3.1a) and (3.2) of the form $A_{1}^{m}(t) B_{1}^{k}(t) \cos \left(N q_{c} r_{1} / 2\right) \cos \left(\ell \sqrt{3} q_{c} r_{2} / 2\right)$. Thus $\sigma$ and $a_{1}$ are given by (2.3) and (2.8) and we need only evaluate the remaining Landau constants $a_{0}$ and $a_{2}$. 
If we proceed in the same manner as we did with the one-dimensional expansion of the last section, the Fredholm-type solvability conditions for $H_{0220}$ and $H_{1220}$, respectively, then yield

$$
\begin{aligned}
a_{0}= & \left(\alpha^{-4}-\alpha^{-10}-\varepsilon+\beta_{c} \alpha^{-1}\right)\left(4 \alpha^{-2}-10 \alpha^{-8}+\beta_{c} \alpha\right) / 8, \\
a_{2}= & \left(13 \alpha^{-4}-19 \alpha^{-10}-9 \varepsilon+10 \beta_{c} \alpha^{-1}\right)\left(10 \alpha^{-9}-4 \alpha^{-3}-\beta_{c}\right) / 16 \\
& +\left(2 \alpha^{-3}+25 \alpha^{-9}+2 \beta_{c}\right)\left(\alpha^{-4}-\alpha^{-10}-\varepsilon+\beta_{c} \alpha^{-1}\right) / 4 .
\end{aligned}
$$

Having determined formulae (2.3), (2.8), and (3.4a,b) for their growth rate and Landau constants, we now return to the six-disturbance hexagonal-planform amplitude-phase equations $(3.1 \mathrm{~b}, \mathrm{c})$. In cataloguing the critical points of these equations and summarizing their orbital stability behavior it is necessary to employ the quantities

$$
\sigma_{-1}=-4 a_{0}^{2} /\left(a_{1}+4 a_{2}\right), \sigma_{1}=16 a_{1} a_{0}^{2} /\left(2 a_{2}-a_{1}\right)^{2}, \sigma_{2}=32\left(a_{1}+a_{2}\right) a_{0}^{2} /\left(2 a_{2}-a_{1}\right)^{2} .
$$

There exist equivalence classes of critical points of (3.1b,c) given by $\phi_{1}=\phi_{2}=\phi_{3}=0$ and I: $A_{1}=A_{2}=A_{3}=0$; II: $A_{1}^{2}=\sigma / a_{1}, A_{2}=A_{3}=0 ; \mathrm{III}^{ \pm}: A_{1}=A_{2}=A_{3}=A_{0}^{ \pm}=\left\{-2 a_{0} \pm\right.$ $\left.\left[4 a_{0}^{2}+\left(a_{1}+4 a_{2}\right) \sigma\right]^{1 / 2}\right\} /\left(a_{1}+4 a_{2}\right) ; \mathrm{IV}: A_{1}=-4 a_{0} /\left(2 a_{2}-a_{1}\right), A_{2}^{2}=A_{3}^{2}=\left(\sigma-\sigma_{1}\right) /\left(a_{1}+2 a_{2}\right) ;$ where it is assumed that $a_{1}, a_{1}+4 a_{2}>0$. The orbital stability conditions for these critical points can be posed in terms of $\sigma$. Thus critical point I is stable in this sense for $\sigma<0$ while the stability behavior of II and III ${ }^{ \pm}$which depends upon the signs of $a_{0}$ and $2 a_{2}-a_{1}$ as well has been summarized in Table 1 under the further assumption that $a_{1}+a_{2}>0$.

TABLE 1

Orbital stability behavior of critical points II and III ${ }^{ \pm}$

\begin{tabular}{ccl}
\hline$a_{0}$ & $2 a_{2}-a_{1}$ & \multicolumn{1}{c}{ Stable structures } \\
\hline+ &,- 0 & $\mathrm{III}^{-}$for $\sigma>\sigma_{-1}$ \\
+ & + & $\mathrm{III}^{-}$for $\sigma_{-1}<\sigma<\sigma_{2}$, II for $\sigma>\sigma_{1}$ \\
0 & - & $\mathrm{III}^{ \pm}$for $\sigma>0$ \\
0 & + & $\mathrm{II}_{\text {for }} \sigma>0$ \\
- & + & $\mathrm{III}^{+}$for $\sigma_{-1}<\sigma<\sigma_{2}$, II for $\sigma>\sigma_{1}$ \\
- &,- 0 & $\mathrm{III}^{+}$for $\sigma>\sigma_{-1}$ \\
\hline
\end{tabular}

In this parameter range $A_{0}^{+}>0$ and $A_{0}^{-}<0$. Finally critical point IV, which reduces to II for $\sigma=\sigma_{1}$ and to $\mathrm{III}^{ \pm}$for $\sigma=\sigma_{2}$ and hence is called a generalized cell, is not stable for any value of $\sigma$.

We next offer a morphological interpretation of the potentially stable critical points catalogued above relative to the thin liquid film patterns under investigation. Then critical points I and II represent the planar films of uniform depth and the surface ridges, respectively, described in the previous section. Observing that to lowest order the layer depth associated with critical points $\mathrm{III}^{ \pm}$ satisfies

$$
H(\mathbf{r}, t)-\alpha \sim A_{0}^{ \pm} f_{0}\left(r_{1}, r_{2}\right)
$$

where

$$
f_{0}\left(r_{1}, r_{2}\right)=\cos \left(2 \pi r_{1} / \lambda_{c}\right)+2 \cos \left(\pi r_{1} / \lambda_{c}\right) \cos \left(\sqrt{3} \pi r_{2} / \lambda_{c}\right)
$$


and noting that the function $f_{0}$ exhibits hexagonal symmetry, we can deduce that these critical points represent hexagonal cellular patterns possessing individual cells with elevated boundaries and depressions at their centers for $\mathrm{III}^{-}$and with elevations at their centers and depressed boundaries for $\mathrm{III}^{+}$. Hence we make the following morphological identifications: $\mathrm{III}^{-}$, a network-like structure of regular hexagonal cells; and $\mathrm{III}^{+}$, a close-packed array of nanodroplets; each of these patterns being separated by relatively flat ultra thin films. Although the latter assertion may seem somewhat conjectural due to our use of weakly nonlinear stability theory, we have confidence in it given that the numerical analyses of similar equations catalogued earlier yielded patterns of precisely this sort as will be described in Section 4.

Having summarized those stability criteria and morphological identifications, we now return to our expressions for the Landau constants of equations $(3.1 \mathrm{~b}, \mathrm{c})$ given by (2.8) and (3.4). First we examine the signs of $a_{0}, 2 a_{2}-a_{1}, a_{1}+4 a_{2}$, and $a_{1}+a_{2}$ as functions of $\alpha$ for $\beta_{c}=0,1,7$ and $\varepsilon=10^{-11}$. Then from the results of this examination we observe that besides $\alpha_{3}$ and $\alpha_{4}$ defined in (2.11) there exist the following other significant values of $\alpha$ :

$$
\alpha_{3}<\alpha_{5}<\alpha_{6}<\alpha_{7}<\alpha_{c}<\alpha_{4}<\alpha_{8}<\alpha_{9}
$$

such that

$$
\begin{gathered}
a_{1}+a_{2}=0 \quad \text { for } \alpha=\alpha_{5} \text { or } \alpha_{8}, \quad a_{1}+a_{2}>0 \quad \text { for } \alpha_{5}<\alpha<\alpha_{8} \\
a_{1}+4 a_{2}=0 \quad \text { for } \alpha=\alpha_{6} \text { or } \alpha_{9}, \quad a_{1}+4 a_{2}>0 \quad \text { for } \alpha_{6}<\alpha<\alpha_{9} \\
a_{0}=0 \text { for } \alpha=\alpha_{c}, \quad a_{0}<0 \text { for } \alpha<\alpha_{c}, \quad a_{0}>0 \text { for } \alpha>\alpha_{c} \\
2 a_{2}-a_{1}=0 \text { for } \alpha=\alpha_{7}, \quad 2 a_{2}-a_{1}<0 \text { for } \alpha<\alpha_{7}, \quad 2 a_{2}-a_{1}>0 \text { for } \alpha>\alpha_{7} .
\end{gathered}
$$

These values of $\alpha$ are compiled in Table 2 .

TABLE 2

The $\alpha$ values of (3.7) versus $\beta_{c}$ for $\varepsilon=10^{-11}$

\begin{tabular}{ccccccc}
\hline$\beta_{c}$ & $\alpha_{5}$ & $\alpha_{6}$ & $\alpha_{7}$ & $\alpha_{c}$ & $\alpha_{8}$ & $\alpha_{9}$ \\
\hline 0 & 1.023 & 1.026 & 1.037 & 1.165 & 1.321 & 1.328 \\
1 & 0.935 & 0.938 & 0.950 & 1.110 & 1.293 & 1.306 \\
7 & 0.805 & 0.808 & 0.821 & 0.988 & 1.187 & 1.209 \\
\hline
\end{tabular}

We again demonstrate our results explicitly for the parameter values of Fig. $3 \mathrm{~b}$ by graphing $a_{1}+$ $4 a_{2}, 2 a_{2}-a_{1}, a_{0}$, and $a_{1}+a_{2}$ versus $\alpha$ in the two parts of Fig. 4 with $\beta_{c}=0$ and $\varepsilon=10^{-11}$. Here we are focusing our attention on this case since the numerical simulations and experimental observations with which the predictions to follow will be compared in the next section are for such an isothermal situation. Given that the stability behavior outlined in Table 1 depends not only on the signs of $a_{0}$ and the various combinations of Landau constants appearing in Fig. 4 but also on the relative sizes of $\sigma, \sigma_{-1}, \sigma_{1}$, and $\sigma_{2}$, we next plot this growth rate and those quantities of (3.5) versus $\alpha$ in Fig. 5 for $\beta_{c}=0$ and $\varepsilon=10^{-11}$.

We are finally ready to make theoretical pattern formation predictions based upon the existence and morphological stability conditions for our critical points summarized earlier. We begin by noting that since critical point I was not stable over any parameter range for the one-dimensional 
(a)

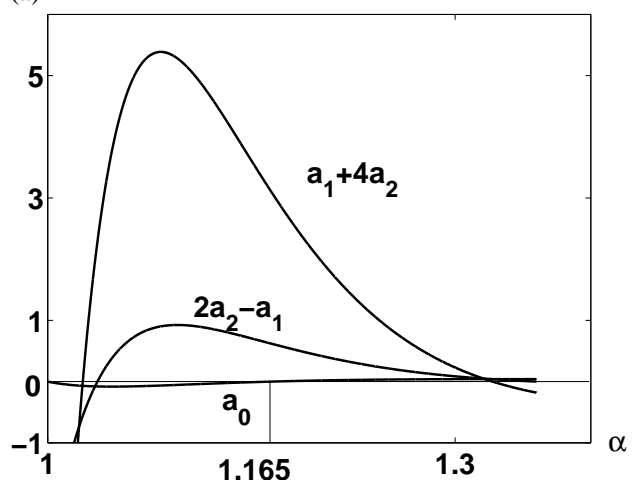

(b)

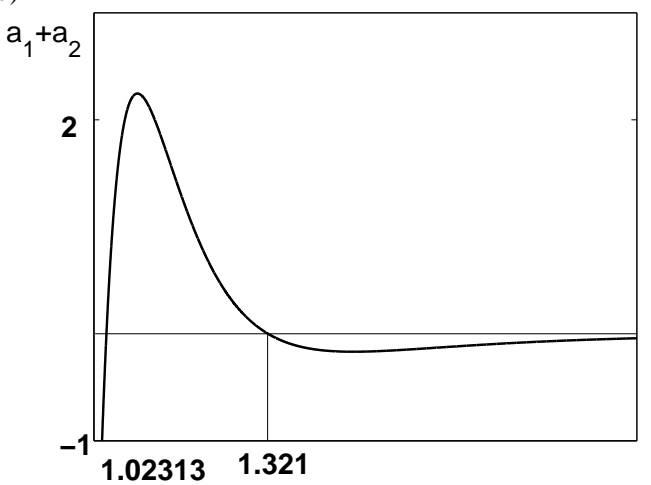

FIG. 4. Plots of (a) $a_{1}+4 a_{2}, 2 a_{2}-a_{1}, a_{0}$, and (b) $a_{1}+a_{2}$ of (2.8) and (3.4) versus $\alpha$ for $\beta_{c}=0$ and $\varepsilon=10^{-11}$.

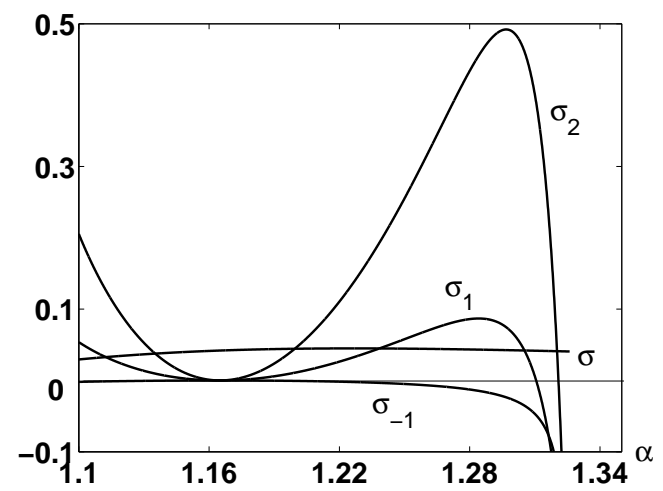

FIG. 5. Plots of $\sigma_{-1}, \sigma_{1}$, and $\sigma_{2}$ of (3.5) as well as $\sigma$ of Fig. 2c versus $\alpha$ for $\beta_{c}=0$ and $\varepsilon=10^{-11}$.

analysis, it is unstable for the hexagonal-planform two-dimensional analysis as well, the latter conclusion following from the one-dimensional perturbation expansion being a special case of the two-dimensional one. Recalling that critical point IV also is never stable, we need only investigate critical points II and $\mathrm{III}^{ \pm}$in this regard. As can be seen from the definitions of these critical points and Table 1 , they must satisfy certain inequality constraints as necessary conditions to yield stable patterns: $a_{1}, 2 a_{2}-a_{1}>0$ for II; $a_{1}+4 a_{2}, a_{1}+a_{2}>0$ and $a_{0}<0$ for III ${ }^{+} ; a_{1}+4 a_{2}, a_{1}+a_{2}, a_{0}>0$ for $\mathrm{III}^{-}$. Hence from Fig. 3b, Table 2, and Fig. 4, we deduce the relevant $\alpha$-interval of interest associated with each critical point: $(1.037,1.311)$ for II; $(1.026,1.165)$ for $\mathrm{III}^{+} ;(1.165,1.321)$ for III $^{-}$. It alone remains to refine these predictions by employing the stability criteria of Table 1 in conjunction with Fig. 5. Toward this end we generate Fig. 6, the six parts of which comprise both an extension and enlargement of Fig. 5 emphasizing those $\alpha$-subintervals that exhibit qualitatively different morphological stability behavior. Let us examine the parts of this figure on a case by case basis as follows:

(a) $\alpha \in(1.026,1.037]: \quad$ Then $a_{0}<0$ and $2 a_{2}-a_{1} \leqslant 0$. Hence, since $\sigma>0 \geqslant \sigma_{-1}$ over the whole range of Fig. 6 (see Fig. 5), we can conclude from the last row of Table 1 that nanodroplets are the 
(a)

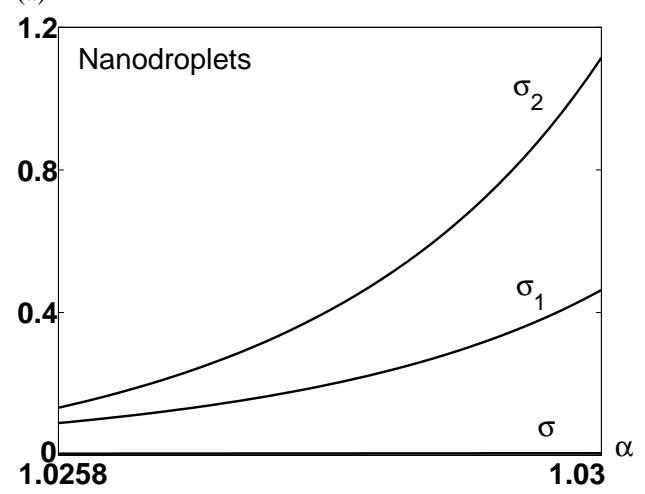

(c)

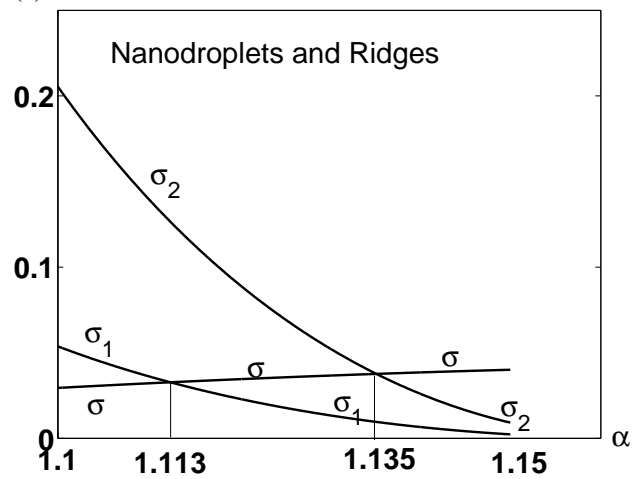

(e)

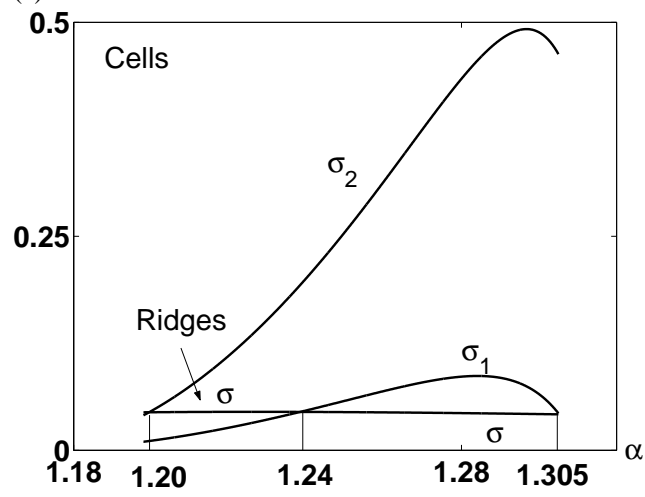

(b)

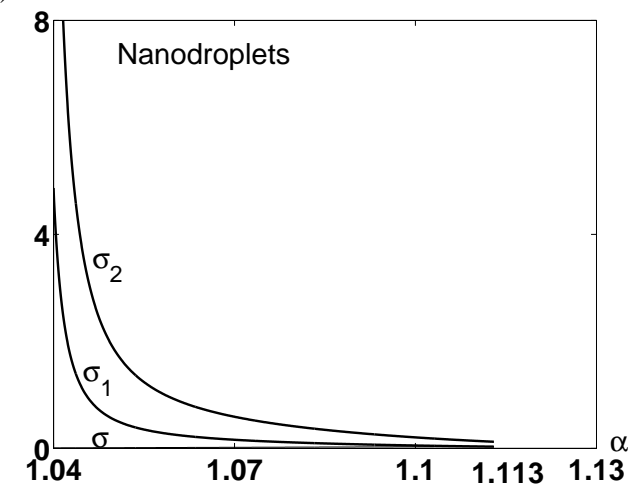

(d)

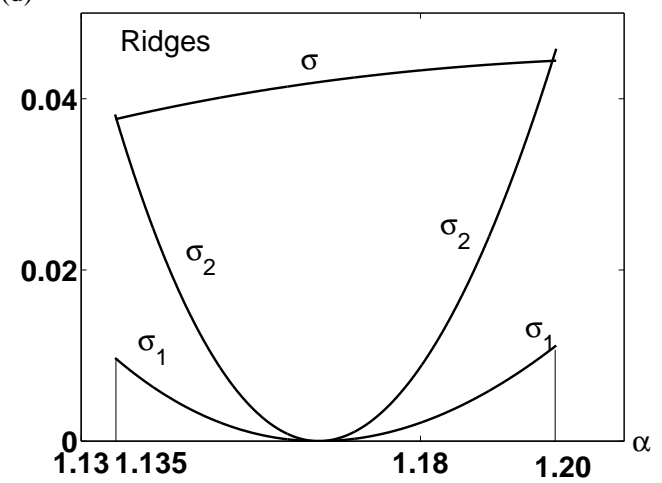

(f)

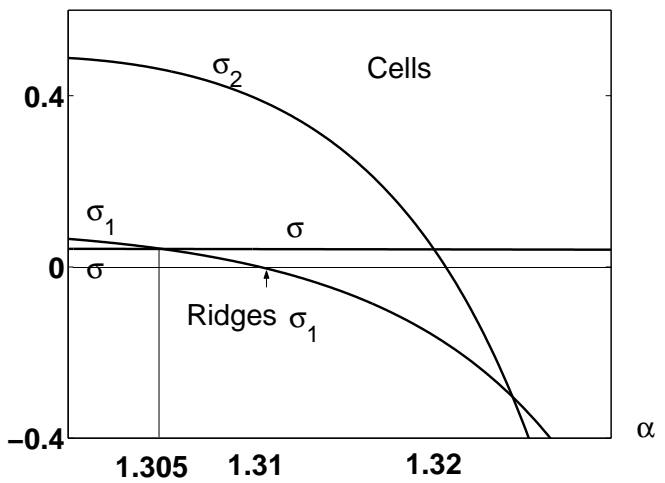

FIG. 6. An extension (a) $\alpha \in(1.026,1.037]$, (b) $\alpha \in(1.037,1.113]$ and enlargement, (c) $\alpha \in(1.113,1.135)$, (d) $\alpha \in$ $[1.135,1.20]$, (e) $\alpha \in(1.20,1.305]$, (f) $\alpha \in(1.305,1.321)$ of the $\sigma, \sigma_{1}$, and $\sigma_{2}$ plots of Fig. 5 indicating the morphological stability predictions catalogued in Table 3 . 
only stable pattern. Note that $\sigma_{1,2}$ play no substantive role in this regime and have only been plotted in Fig. 6a for the sake of completeness.

(b) $\alpha \in(1.037,1.113]$ : Then $a_{0}<0$ and $2 a_{2}-a_{1}>0$. Hence, since $0<\sigma \leqslant \sigma_{1}<\sigma_{2}$, we can conclude from the fifth row of Table 1 that nanodroplets are the only stable pattern.

(c) $\alpha \in(1.113,1.135): \quad$ Then $a_{0}<0$ and $2 a_{2}-a_{1}>0$. Hence, since $0<\sigma_{1}<\sigma<\sigma_{2}$, we can conclude from the fifth row of Table 1 that there is bistability between nanodroplets and ridges.

(d) $\alpha \in[1.135,1.20]: \quad$ We shall further partition this case into the three subcases:

(i) $\alpha \in[1.135,1.165): \quad T h e n a_{0}<0$ and $2 a_{2}-a_{1}>0$. Hence, since $0<\sigma_{1}<\sigma_{2} \leqslant \sigma$, we can conclude from the fifth row of Table 1 that ridges are the only stable pattern.

(ii) $\alpha=1.165$ : Then $a_{0}=0$ and $2 a_{2}-a_{1}>0$. Hence, since $\sigma>0=\sigma_{1}=\sigma_{2}$, we can conclude from the fourth row of Table 1 that ridges are the only stable pattern.

(iii) $\alpha \in(1.165,1.20]$ : Then $a_{0}>0$ and $2 a_{2}-a_{1}>0$. Hence, since $0<\sigma_{1}<\sigma_{2} \leqslant \sigma$, we can conclude from the second row of Table 1 that ridges are the only stable pattern.

Taken together these subcases yield the result that ridges are the only stable pattern in this whole subinterval.

Note that for the rest of the cases to be catalogued below, $a_{0}, 2 a_{2}-a_{1}>0$ as in the subcase just examined and thus we shall employ the second row of Table 1.

(e) $\alpha \in(1.20,1.305]$ : We shall further partition this case into the two subcases:

(i) $\alpha \in(1.20,1.24)$ : Hence, since $0<\sigma_{1}<\sigma<\sigma_{2}$, we can conclude that there is bistability between ridges and cells.

(ii) $\alpha \in[1.24,1.305]$ : Hence, since $0<\sigma \leqslant \sigma_{1}<\sigma_{2}$, we can conclude that cells are the only stable pattern.

(f) $\alpha \in(1.305,1.321):$ We shall further partition this case into the three subcases:

(i) $\alpha \in(1.305,1.311)$ : Hence, since $0<\sigma_{1}<\sigma<\sigma_{2}$, we can conclude that there is bistability between cells and ridges.

(ii) $\alpha \in[1.311,1.32)$ : Then $a_{1} \leqslant 0$. Hence, since $\sigma_{1} \leqslant 0<\sigma<\sigma_{2}$, we can conclude that cells are the only stable pattern because ridges do not exist.

(iii) $\alpha \in[1.32,1.321)$ : Then $a_{1}<0$. Hence, since $\sigma_{1}<0<\sigma_{2} \leqslant \sigma$, we can conclude that there are no stable patterns because ridges do not exist and cells are unstable.

These morphological stability predictions are summarized in Table 3.

Observe from Fig. 4a that

$$
\left|a_{0}\right| \ll a_{1}+4 a_{2}
$$

in the parameter range of interest and thus $\sigma_{-1}$ although negative is virtually indistinguishable from zero over much of that range (see Fig. 5). Further to justify the truncation procedure inherent to the asymptotic representation of (3.1) it is necessary that the Landau constants of its amplitude-phase equations satisfy the additional size constraint [32]

$$
\left|a_{0}\right| /\left(a_{1}+4 a_{2}\right)^{2} \ll 1 .
$$

Noting that the inequality condition (3.8a) also guarantees the satisfaction of this constraint, we can conclude that such a truncation procedure is valid for our hexagonal-planform weakly nonlinear stability analysis of the thin liquid film model system under investigation. 
TABLE 3

Morphological stability predictions versus $\alpha$

\begin{tabular}{ll}
\hline \multicolumn{1}{c}{$\alpha$ range } & \multicolumn{1}{c}{ Stable pattern } \\
\hline$(1.026,1.113]$ & Nanodroplets \\
$(1.113,1.135)$ & Nanodroplets \& Ridges \\
{$[1.135,1.20]$} & Ridges \\
$(1.20,1.24)$ & Ridges \& Cells \\
{$[1.24,1.305]$} & Cells \\
$(1.305,1.311)$ & Cells \& Ridges \\
{$[1.311,1.32)$} & Cells \\
\hline
\end{tabular}

\section{Comparisons, discussion, and conclusions}

To facilitate comparison of our morphological stability predictions with the results of the pattern formation studies involving thin liquid films referenced earlier, we describe those theoretical and experimental outcomes in more detail. The three photographs of different morphologies exhibited by unstable thin polymer films appearing in Reiter [19] depict an undulation pattern and the early and late stages of cellular pattern formation in which the elevated rims of the initial circular cylindrical depressions coalesce to form network-like regular hexagonal cells of nearly identical size. In previous work Reiter [18] described that pattern as a polygonal cellular structure where the most often found polygon was a hexagon with its vertices joining three edges and these hexagons were of uniform size by virtue of the coalescence process of the elevated rims of the initial depressions. The morphologies exhibited by these stages are precisely the topographies expected for type $\mathrm{III}^{-}$structures; hence, our identification of the latter with cells. Let us turn to the other morphology described by Reiter [19], the undulation pattern. We have identified such patterns with surface ridges characteristic of type II structures. This identification is consistent with Oron and Bankoff's [15] theoretical prediction for a one-dimensional weakly nonlinear stability analysis of their evolution equation. There also exist numerical simulations of one-dimensional versions of such equations which produced stationary solutions by Sharma and Jameel [22], Mitlin and Petviashvilli [14], and Oron and Bankoff [15]. Restricting their analyses to a single disturbance wavelength, they found a stationary arch-type solution for certain layer thicknesses. Mitlin and Petviashvilli [14] and Oron and Bankoff [15] interpreted this solution as a one-dimensional spatially periodic stable liquid ridge-type pattern while Sharma and Jameel [22] made the interpretation that it represented nanodroplets separated by relatively flat ultra thin films. In deciding between these interpretations it is instructive to point out Reiter's [19] having reported that none of his experimental states represented an equilibrium situation of isolated droplets. Although we feel that isolated nanodroplets do not represent the best identification of stationary one-dimensional periodic patterns, a uniform close-packed distribution of such droplets referred to as morphological phase separation by Sharma and Jameel [22] and Jameel and Sharma [6] is consistent with the topography of type $\mathrm{III}^{+}$structures for thin liquid films. Hence we have identified the $\mathrm{III}^{+}$critical point with morphological phase separation of this sort.

Returning to the parallel surface ridges predicted by one-dimensional nonlinear stability analyses and numerical simulations, Khanna and Sharma [8] point out that such regular arrangements of cylindrical hills and valleys represent a pattern never witnessed in thin liquid film 
experiments. Instead interconnected bicontinuous patterns are observed. Since the equivalence class of critical points designated as II in the last section actually contains the three solutions [20]

$$
A_{i}^{2}=\sigma / a_{1}, \quad A_{j}=A_{k}=0, \quad(i, j, k)=\text { even permutation of }(1,2,3)
$$

each of the $\alpha$-intervals of Table 3 identified with ridges is itself a locus of multiple stable states. These represent a family of ridges aligned parallel to the $r_{2}$-axis as per our original identification, plus two similar families of ridges making angles of $\pm 60^{\circ}$ to them for which stable co-existence with a member of either the original family or one another is impossible [20]. Then, as initial conditions vary from point to point on the interfacial surface, such families of ridges can give rise to polygonal arcs the boundaries of which would appear quite random in orientation. Indeed the interconnected bicontinuous pattern classified as an undulation by Reiter [19] has the appearance of such curved elongated ridges in the relevant photographic reproduction contained therein.

We are now ready to compare our two-dimensional morphological stability predictions with the isothermal numerical simulations and experimental observations mentioned previously. Sharma and Khanna [23] performed two-dimensional numerical simulations on an equation analogous to (1.12) for $\varepsilon=\beta=0$ but with the repulsive force term of (1.6a) taken proportional to $\exp \left(-h / \ell_{0}\right)$ with the correlation length $\ell_{0}=2.5 \mathrm{~nm}$. They found two completely different morphological patterns by which pseudo-dewetting - i.e., elevations separated by ultra thin flat films-could occur based upon the mean layer thickness. For relatively thick films the rims of the circular depressions which formed first from an initial bicontinuous pattern developed into a polygonal structure by repeated coalescence. In contrast to this scenario for relatively thin films the bicontinuous pattern fragmented directly to produce a uniform array of microdroplets. For a range of intermediate thickness the bicontinuous structure composed of long hills and valleys resolved itself into a mixture with these other two patterns, the type and proportion of which depended, in our notation, on the relative distance $h_{0}-h_{c}$.

Xie et al. [36] investigated true dewetting of polystyrene films on a silicon substrate at a fixed annealing temperature $T \equiv T_{0}=388^{\circ} \mathrm{K}$ as a function of mean layer thickness in the range $h_{0} \in$ [4.5 nm, $35 \mathrm{~nm}$ ]. For $h_{0}=7.5 \mathrm{~nm}$ they observed a bicontinuous surface pattern while for $h_{0}=$ $12.5 \mathrm{~nm}$ they saw a bistable state between configurations resembling Voronoi tessellation patterns and these surface undulations. For even thinner films such as $h_{0}=4.5 \mathrm{~nm}$ the initial bicontinuous pattern broke up into small uniformly distributed droplets that subsequently coarsened to form large isolated drops by coalescence and hence dewetted the surface. They contrasted this behavior to the dewetting of much thicker films such as $h_{0}=35 \mathrm{~nm}$ in which rupture occurred by the formation of circular holes.

From the two-dimensional morphological stability predictions of Table 3 in conjunction with the one-dimensional results of Section 2, we can draw the following general conclusions: Although the planar interface solution is never stable, supercritical equilibrium patterns occur for an interval of mean layer thickness $\alpha \in(1.026,1.32)$ with subcritical rupture occurring outside this interval. These patterns consist of bicontinuous surface ridges and hexagonal network-like cells or closepacked configurations of nanodroplets separated by relatively flat ultra thin films. In particular those morphological phase separation patterns are crucially dependent upon the inclusion of the short-range intermolecular repulsive force with cells tending to be stable for the thicker layers; nanodroplets, for the thinner ones; and ridges, for layers of intermediate thickness. These theoretical predictions are in qualitative accord with both the relevant isothermal experimental evidence and numerical simulations of similar model equations cited above as well as being consistent with 
dewetting-type rupture occurring for such situations by hole formation in relatively thick layers but by drop formation in thinner ones.

In order to make these qualitative comparisons of theoretical prediction with experimental evidence and numerical simulation more quantitative in nature, we next examine the effect on our results of taking a different choice for $h_{c}$, this critical thickness being a measure of the coupling between the long-range attractive and short-range repulsive intermolecular forces. Recall from (1.12c) that $h_{0}$ is related to $\alpha$ by

$$
h_{0}=\alpha h_{c}
$$

and $\alpha=\alpha_{c}$ corresponds to the crossover value of mean layer thickness at which the morphology switches its character. Further note that when $\beta_{c}=0, h_{c}$ only influences our problem by its presence in the dimensionless parameter $\varepsilon$ which may be rewritten as

$$
\varepsilon=\left(h_{c} / h_{g}\right)^{4} \quad \text { where } \quad h_{g}=\left(\frac{3 a}{\rho_{0} g}\right)^{1 / 4} .
$$

We observe in this context that the results of our one-dimensional problem are extremely robust with respect to changes in $\varepsilon$. In particular $\alpha_{1}, \alpha_{3}$, and $\alpha_{4}$ are virtually invariant as $\varepsilon$ ranges over a wide range of values since Fig. 3a is altered very little during this process. Then, although $\alpha_{2}$ varies drastically with $\varepsilon$ since $\alpha_{2} \sim \varepsilon^{-1 / 4}$ as $\varepsilon \rightarrow 0$ when $\beta_{c}=0$, observe that the asymptotic representation for the corresponding dimensional thickness

$$
h_{2}=\alpha_{2} h_{c} \cong \varepsilon^{-1 / 4} h_{c}=h_{g}
$$

is actually independent of $h_{c}$. Similarly the results of our two-dimensional problem are also robust with respect to changes in $\varepsilon$. Hence, to determine the isothermal predictions of our problem for different $h_{c}$, we need only employ the significant values of $\alpha$ for $h_{c}=1 \mathrm{~nm}$ from Table 3 in conjunction with (4.2). For example by taking

$$
h_{c}=7.725 \mathrm{~nm},
$$

we would deduce a crossover layer depth related to $\alpha_{c}=1.165$ of

$$
h_{c r}=\alpha_{c} h_{c}=9 \mathrm{~nm}
$$

in quantitative agreement with the observations of Xie et al. [36] and the simulations of Sharma and Khanna [23]. For the $h_{c}$ value of (4.4a) our patterned layer interval $\alpha \in(1.026,1.32)$ corresponds to $h_{0} \in(7.926 \mathrm{~nm}, 10.197 \mathrm{~nm})$ while $\varepsilon=3.56 \times 10^{-8}$ and the upper instability bound is retained at $h_{g}=562 \mathrm{~nm}$.

In the same vein we develop an expression for the dimensional wavelength $\lambda_{c}^{*}$ which from the length scale factors of (1.10) and (1.11) is related to $\lambda_{c}$ of (2.12) by

$$
\lambda_{c}^{*}=h_{c}(S / A)^{1 / 2} \lambda_{c}=h_{c}^{2}\left(\frac{\gamma_{0}}{3 a}\right)^{1 / 2} \frac{2 \pi}{q_{c}} .
$$

For $\beta_{c}=0$,

$$
1 / q_{c} \sim\left(\alpha^{-4}-\alpha^{-10}\right)^{-1 / 2} \quad \text { as } \varepsilon \rightarrow 0
$$

while

$$
\left(\alpha^{-4}-\alpha^{-10}\right)^{-1 / 2} \sim \alpha^{2} \quad \text { when } \alpha \gg 1 .
$$


Then, observing that with $\alpha=2$ the left- and right-hand sides of this relation yield the results 4.03 and 4, respectively, we substitute (4.6) into (4.5) and obtain

$$
\lambda_{c}^{*} \sim 2 \pi\left(\frac{\gamma_{0}}{3 a}\right)^{1 / 2}\left(h_{c} \alpha\right)^{2}=2 \pi\left(\frac{\gamma_{0}}{3 a}\right)^{1 / 2} h_{0}^{2},
$$

which upon taking the typical value $\gamma_{0}=50$ dynes/cm [25] in conjunction with (2.5), becomes

$$
\lambda_{c}^{*} \sim 10 \sqrt{2} \pi h_{0}^{2} / \mathrm{nm} .
$$

Note that this asymptotic expression is independent of $h_{c}$ and consistent with the results of Xie $e t$ al. [36] and Sharma and Khanna [23] who both deduced that $\lambda_{c}^{*}$ was proportional to $h_{0}^{2}$ as well as with those of Bischof et al. [1] whose thin liquid metal film patterns exhibited the same relationship.

Having examined the effect of varying $\varepsilon$ on our model equation, we turn to its $\beta_{c}$-dependence. Unlike many Marangoni-type convection problems [3], increasing $\beta_{c}$ from zero in ours only has the quantitative effect of decreasing all the significant $\alpha$ values of Fig. 3a and Table 2 rather than producing any qualitatively different stability behavior. Also unlike most other such BénardMarangoni model systems, our thin liquid film problem satisfies the truncation criterion (3.8b). As Thess and Orszag [27] pointed out, although bifurcation theory has significantly improved our understanding of nonlinear convection, a mathematical inconsistency remained present in all applications of this theory to actual Bénard-Marangoni model systems developed up to that time; namely, the violation of condition (3.8) caused by

$$
a_{0}=O\left(a_{1}+4 a_{2}\right)=O(1) .
$$

Davis [3] noted that the results of such a hexagonal-planform analysis of these model systems can then be merely considered suggestive at best. We finish this discussion by observing that even in the isothermal case of $\beta_{c}=0$ it was still necessary to include the Marangoni effect during our evolution equation derivation in order subsequently to employ the approach of Sekimura et al. [21].

It only remains for us to place our thin liquid film results in the context of some recent pattern formation studies. We begin this commentary with a comparison of our morphological stability predictions to equilibrium structures characteristic of systems used to model chemical Turing pattern formation in gel reactors. Wollkind and Stephenson [34] investigated the development of one- and two-dimensional Turing patterns observed in chlorite-iodide-malonic acid (CIMA)/indicator gel reactor experiments by performing both rhombic and hexagonal planform weakly nonlinear stability analyses on the appropriately scaled general reaction-diffusion activator-inhibitor/immobilizer model system of Stephenson and Wollkind [26]. Then they particularized their results to the Lengyel and Epstein [11, 12] chlorine dioxide-iodine-malonic acid (CDIMA)/indicator model system used to represent this chemical reaction. The stable critical points of the hexagonal-planform amplitudephase equations which can potentially have a variety of phenomenological interpretations in this instance corresponded to the following Turing instability distribution patterns: I, homogeneous; II, stripes; $\mathrm{III}^{+}$, a hexagonal net-like pattern of relatively low iodide concentration or honeycombs; and III $^{-}$, a hexagonal close-packed array of nearly-circular dot-like regions of relatively low iodide concentration or spots. Since these Turing patterns have been characterized by low iodide concentration the roles of the honeycombs and spots of that phenomenon have been reversed from those of the nanodroplets and cells of our thin liquid film model where the morphologies are formed by surface elevations. The Landau constants for this chemical Turing pattern problem satisfied 
relationships similar to the ones of (3.7) and (3.8) when $\alpha$ was identified with a nondimensional malonic acid concentration except that (3.7a) and (3.7e) were replaced by the more symmetric

$$
\alpha_{3}<\alpha_{5}<\alpha_{6}<\alpha_{7}<\alpha_{c}<\alpha_{10}<\alpha_{9}<\alpha_{8}<\alpha_{4}
$$

and

$$
\begin{aligned}
& 2 a_{2}-a_{1}=0 \quad \text { for } \alpha=\alpha_{7} \text { or } \alpha_{10}, \quad 2 a_{2}-a_{1}>0 \quad \text { for } \alpha_{7}<\alpha<\alpha_{10}, \\
& 2 a_{2}-a_{1}<0 \quad \text { for } \alpha<\alpha_{7} \text { or } \alpha>\alpha_{10}
\end{aligned}
$$

respectively. Hence the $\alpha$-interval of interest for this chemical Turing pattern problem was $\alpha \in$ $\left(\alpha_{6}, \alpha_{9}\right)$. Note that for our problem with $\varepsilon=10^{-11}$ and $\beta_{c}=0, \alpha_{10}=1.36>\alpha_{9}>\alpha_{8}$ and thus did not have to be considered explicitly. From those results Wollkind and Stephenson [34] were able to determine the parameter range corresponding to the Turing patterns catalogued above and found that for $a_{1}+4 a_{2}>0$ all (when $2 a_{2}-a_{1} \leqslant 0$ ) or part (when $2 a_{2}-a_{1}>0$ ) of the parameter space where Stephenson and Wollkind [26] predicted stripes was further divided into two subspaces characterized by hexagonal patterns consisting of either spots (when $a_{0}>0$ ) or honeycombs (when $a_{0}<0$ ) with $a_{0}=0$ for $\alpha=\alpha_{c}=1.88$. They then compared these Turing pattern predictions with both CIMA/indicator experimental observations and theoretical results relevant to the analogous morphological stability analysis of the prototype alloy solidification problem of Wollkind et al. [33]. In the former instance that comparison was quite favorable while in the latter it was very similar when $\alpha$ was identified with a dimensionless rate of solidification. In particular the stable critical points had the following solid-liquid interfacial identifications: I, planar interface; II, bands; $\mathrm{III}^{+}$, dome-shaped regular hexagonal cells or nets; and $\mathrm{III}^{-}$, a hexagonal close-packed array of circular depressions of liquid into the solid or nodes. Indeed Wollkind and Stephenson [35] actually exploited this similarity when offering their suggested explanation for the black-eye Turing patterns of Ouyang and Swinney [17] as being a superposition of spots and honeycombs occurring on the top and bottom of the thin patterned layer. In this context we note that a number of the Turing patterns classified as stripes by Ouyang and Swinney [17] in their relevant photographic reproductions bear a striking resemblance to our bicontinuous ridges.

Contemporaneously Judd and Silber [7] extended this six-disturbance hexagonal-planform analysis of the Lengyel and Epstein CDIMA model system to a twelve-disturbance superlattice one developed by Dionne et al. [4]. That approach allowed them to investigate the possibility of occurrence of both our simple patterns periodic on a length scale of the critical wavelength of linear theory and superlattice patterns periodic on a much longer scale. They found that unless $a_{0}=0$ all patterns bifurcated unstably and unfolding this degeneracy by considering $0<\left|a_{0}\right| \ll 1$ obtained morphological stability predictions involving only simple periodic patterns consistent with those of Wollkind and Stephenson [34]. Given the dependence of the experimentally observed thin liquid film patterns on $\lambda_{c}^{*}$ and the similarity in $a_{0}$ behavior between the CDIMA model system and evolution equation (1.12), the six-disturbance hexagonal-planform analysis of it presented in the last section would seem to be sufficient for our purposes.

Since the existence of an $\alpha=\alpha_{c}$ at which $a_{0}$ vanishes plays such a fundamentally important part in our morphological stability predictions, there is some merit in examining this behavior more thoroughly. From (2.3b) and (3.4a) we see that $a_{0}$ can be represented in the form

$$
a_{0}=Q_{c}\left(\alpha ; \beta_{c}, \varepsilon\right)\left(4 \alpha^{-2}-10 \alpha^{-8}+\beta_{c} \alpha\right) / 8
$$


and hence conclude that $\alpha_{c}$ satisfies

$$
4 \alpha_{c}^{-2}-10 \alpha_{c}^{-8}+\beta_{c} \alpha_{c}=0,
$$

which is independent of $\varepsilon$ and yields the explicit solution for $\beta_{c}=0$ of

$$
\alpha_{c}=(5 / 2)^{1 / 6} \cong 1.165
$$

Then (4.10b) in conjunction with (2.8) and (3.4b) implies that

$$
a_{1}=a_{2} \quad \text { for } \alpha=\alpha_{c}
$$

or

$$
2 a_{2}-a_{1}=a_{1}=Q_{c}\left(\alpha_{c} ; \beta_{c}, \varepsilon\right)\left(2 \alpha_{c}^{-3}+25 \alpha_{c}^{-9}+2 \beta_{c}\right) / 4>0 .
$$

Thus the vanishing of the quadratic terms in the amplitude-phase equations guarantees that ridges alone but never hexagonal patterns can be stable for our thin liquid film problem. Therefore in spite of the potentiality of bistability existing between the two types of hexagonal states when $a_{0}=0$ and $2 a_{2}-a_{1}<0$ (see the appropriate entry of Table 1 which actually only refers to neutrally stable structures in this instance) that particular possibility is precluded for our specific model system.

We conclude with a final comparison between our one-dimensional results and those obtained if the repulsive force term used by Oron and Bankoff [15] is employed. Recall that the latter authors felt their repulsive potential term proportional to $h^{-4}$ was superior to one proportional to $h^{-9}$. We wish to explore this matter further by comparing the morphological stability predictions corresponding to each of these choices for the repulsive potential. Toward that end we consider the intermolecular force potential

$$
\phi(h)=\left(a / h_{c}^{3}\right)\left[\left(h_{c} / h\right)^{3}-3\left(h_{c} / h\right)^{4} / 4\right]
$$

instead of (1.9a) and produce an evolution equation which differs from (1.12) only in the replacement of its $H^{-7}$ term by $H^{-2}$. Next we perform the same one-dimensional weakly nonlinear stability analysis of Section 2 on that equation and obtain a growth rate still satisfying

$$
\sigma=\alpha^{3} Q_{c}^{2}\left(\alpha ; \beta_{c}, \varepsilon\right) / 4
$$

but now with

$$
Q_{c}\left(\alpha ; \beta_{c}, \varepsilon\right)=\alpha^{-4}-\alpha^{-5}+\beta_{c} \alpha^{-1}-\varepsilon
$$

and a Landau constant formula

$$
\begin{aligned}
a_{1}= & \left(22 \alpha^{-4}-23 \alpha^{-5}-18 \varepsilon+19 \beta_{c} \alpha^{-1}\right)\left(5 \alpha^{-4}-4 \alpha^{-3}-\beta_{c}\right) / 24 \\
& +\left(\alpha^{-3}+\beta_{c}\right)\left(\alpha^{-4}-\alpha^{-5}-\varepsilon+\beta_{c} \alpha^{-1}\right) / 2 .
\end{aligned}
$$

Then in the isothermal case of $\beta_{c}=0$ and $\varepsilon=10^{-11}$ we can deduce from (4.13) and (4.14) that for $\alpha \in\left(\alpha_{3}, \alpha_{4}\right)$ where $\alpha_{3}=1.027$ and $\alpha_{4}=1.47$,

$$
0<\sigma \leqslant .0036, \quad 0<a_{1} \leqslant .037 .
$$

Given the relative sizes of the upper bounds for these quantities in comparison to those of (2.13) we observe that the truncation procedure inherent to our development in Section 2 will not be as 
simple to implement in this instance. Hence we feel that the form of our repulsive potential force term is more appropriate for the purpose of weakly nonlinear stability theory than the one chosen by Oron and Bankoff [15]. We now reiterate a point made above about Bénard-Marangoni model systems. Weakly nonlinear stability analyses of this type do not necessarily apply to all patternforming systems but only hold under certain restricted conditions that need to be established on a case by case basis.

In summary this thin liquid film problem, involving a single evolution equation, is compatible with our long range aim of developing the simplest reasonable natural science models which preserve the essential features of pattern generation and are still consistent with observation. We believe that a procedure which employs analytical stability techniques to establish the parameter range of interest for pattern formation such as our weakly nonlinear methods and only then examines this regime more fully numerically is advantageous both scientifically and economically to one which attempts to use numerical techniques alone in order to accomplish the same end.

We close by offering an additional rationale for our research. The study of long-wavelength hydrodynamic instabilities generated by intermolecular forces in thin films has a wide variety of applications ranging from industrial processes to biological phenomena all of which involve a liquid layer coating a solid substrate. The development of various models of thin liquid films to be analyzed for their stability such as our prototype evolution equation (1.12) has been motivated by the desire to provide a better understanding of these diverse applications. Reiter [19] stated that Herminghaus et al.'s [5] major achievement was their demonstration that the same coupling mechanism between Van der Waals attractive and Born repulsive forces could account for the different patterns produced in a given experiment and their subsequent suggestion that the exact form of the associated intermolecular potential could be inferred from knowledge of interfacial morphology together with layer thickness as an inverse problem. He then declared that unfortunately it was still unclear why almost the same patterns can be found in both polystyrene and gold films. The primary reason for our thin liquid film research is to answer questions of this sort by explaining more completely the pattern formational behavior of (1.12).

\section{REFERENCES}

1. Bischof, J., Scherer, D., Herminghaus, S. \& Leiderer, P. Dewetting modes of thin metallic films: Nucleation of holes and spinodal dewetting. Phys. Rev. Lett. 77 (1996), 1536-1539.

2. CAhn, J. W. \& Hilliard, J. E. Free energy of a nonuniform system. I. Interfacial free energy. J. Chem. Phys. 28 (1958), 258-267.

3. Davis, S. H. Thermocapillary instabilities. Ann. Rev. Fluid Mech. 19 (1987), 403-435.

4. Dionne, B., Silber, M. \& Skeldon, A. C. Stability results for steady, spatially periodic planforms. Nonlinearity 10 (1997), 321-353. Zbl 0907.58050|MR 98a:58122

5. Herminghaus, S., Jacobs, K., Mecke, K., Bischof, J., Fery, A., Ibn-elhaj, M. \& SCHLAGOWSKI, S. Spinodal dewetting in liquid crystal and liquid metal films. Science 282 (1998), 916919.

6. Jameel, A. T. \& Sharma, A. Morphological phase separation in thin liquid films II. Equilibrium contact angles of nanodrops coexisting with thin films. J. Colloid Interface Sci. 164 (1994), 416-427.

7. JudD, S. L. \& Silber, M. Simple and superlattice Turing patterns in reaction-diffusion systems: bifurcation, bistability, and parameter collapse. Physica D 136 (2000), 45-65.Zbl 0947.35082 MR 2001a:37125

8. Khanna, R. \& Sharma, A. Pattern formation in spontaneous dewetting of thin apolar films. J. Colloid Interface Sci. 195 (1997), 42-50. 
9. Kheshgi, H. S. \& SCRiven, L. E. Dewetting: Nucleation and growth of dry regions. Chem. Engrng. Sci. 46 (1991), 519-526.

10. Koschmieder, E. L. Bénard Cells and Taylor Vortices. Cambridge Univ. Press, Cambridge (1993). Zbl 0780.76003 MR 94h:76031

11. LENGYEL, I. \& EPSTEIN, I. R. Modeling of Turing structures in the chlorite-iodide-malonic acid-starch reaction system. Science 251 (1991), 650-652.

12. Lengyel, I. \& EPSTEIN, I. R. A chemical approach to designing Turing patterns in reaction-diffusion systems. Proc. Nat. Acad. Sci. U.S.A. 89 (1992), 3977-3979. Zbl 0745.92002

13. Mithin, V. S. Dewetting of solid surface: Analogy with spinodal decomposition. J. Colloid Interface Sci. 156 (1993), 491-497.

14. Mitlin, V. S. \& Petviashvili, P. V. Nonlinear dynamics of dewetting: kinetically stable structures. Phys. Lett. A 192 (1994), 323-326.

15. Oron, A. \& BANKoff, S. G. Dewetting of a heated surface by an evaporating liquid film under conjoining/disjoining pressures. J. Colloid Interface Sci. 218 (1999), 152-166.

16. Oron, A., Davis, S. H. \& Bankoff, S. G. Long-scale evolution of thin films. Rev. Mod. Phys. 69 (1997), 931-980.

17. OuYang, Q. \& Swinney, H. L. Onset and beyond Turing pattern formation. Chemical Waves and Patterns, R. Kapral and K. Showalter (eds.), Kluwer, Dordrecht (1995), 269-295.

18. ReIter, G. Dewetting of thin polymer films. Phys. Rev. Lett. 68 (1995), 75-78.

19. ReIter, G. The artistic side of intermolecular forces. Science 282 (1998), 888-889.

20. SEgEL, L. A. The nonlinear interaction of a finite number of disturbances in a layer of fluid heated from below. J. Fluid Mech. 21 (1965), 359-384. Zbl 0244.76024

21. Sekimura, T., Zhu, M., Cook, J., Maini, P. K. \& Murray, J. D. Pattern formation of scale cells in Lepidoptera by differential origin-dependent cell adhesion. Bull. Math. Biol. 61 (1999), 807-827.

22. Sharma, A. \& JAmeel, A. T. Nonlinear stability, rupture, and morphological phase separation of thin fluid films on apolar and polar substrates. J. Colloid Interface Sci. 161 (1993), 190-208.

23. Sharma, A. \& Khanna, R. Pattern formation in unstable thin liquid films. Phys. Rev. Lett. 81 (1998), 3463-3466.

24. Sharma, A. \& Reiter, G. Instability of thin polymer films on coated substrates: Rupture, dewetting, and drop formation. J. Colloid Interface Sci. 178 (1996), 383-399.

25. Sharma, A. \& Ruckenstein, E. An analytical nonlinear theory of thin film rupture and its application to wetting films. J. Colloid Interface Sci. 113 (1986), 456-479.

26. STEPHENSON, L. E. \& WOLLKIND, D. J. Weakly nonlinear stability analyses of one-dimensional Turing pattern formation in activator-inhibitor/immobilizer model systems. J. Math. Biol. 33 (1995), 771-815. Zbl 0832.92003|MR 97a:92004

27. Thess, A. \& OrsZag, S. A. Surface-tension-driven Bénard convection at infinite Prandtl number. J. Fluid Mech. 283 (1995), 201-230. Zbl 0836.76034|MR 95i:76097

28. Tian, M. Pattern formation analyses of thin liquid films. Ph.D. thesis, Washington State Univ. (2001).

29. Van Hook, S. J., Schatz, M. F., Mccormick, W. D., Swift, J. B. \& Swinney, H. L. Longwavelength instability to surface-tension-driven Bénard convection. Phys. Rev. Lett. 75 (1995), 4397-4400.

30. Williams, M. B. \& Davis, S. H. Nonlinear theory of film rupture. J. Colloid Interface Sci. 90 (1982), 220-228.

31. Wollkind, D. J. Rhombic and hexagonal weakly nonlinear stability analyses: Theory and application. Nonlinear Instability Analysis Vol. II, L. Debnath (ed.), WIT Press, Southampton (2001,) 221-272. MR 1890393

32. Wollkind, D. J., Manoranjan, V. S. \& Zhang, L. Weakly nonlinear stability analyses of prototype reaction-diffusion model equations. SIAM Rev. 36 (1994), 176-214. Zbl 0808.35056 MR 95d:35090 
33. Wollkind, D. J., SRiranganathan, R. \& Oulton, D. B. Interfacial patterns during plane front alloy solidification. Physica D 12 (1984), 215-240.

34. Wollkind, D. J. \& Stephenson, L. E. Chemical Turing pattern formation analyses: Comparison of theory with experiment. SIAM J. Appl. Math. 61 (2000), 387-431.Zbl 0992.92002|MR 2001g:76062

35. Wollkind, D. J. \& Stephenson, L. E. Chemical Turing patterns: A model system of a paradigm for morphogenesis. Mathematical Models for Biological Pattern Formation, H. G. Othmer and P. K. Maini (eds.), Springer, Berlin (2001), 113-142.

36. Xie, R., Karim, A., Douglas, J. F., Han, C. C. \& Weiss, R. A. Spinodal dewetting of thin polymer films. Phys. Rev. Lett. 81 (1998), 1251-1254. 